\title{
Uncompetitive Phospholipase A2 Inhibition by CHEC Sequences Including Oral Treatment of Experimental Autoimmune Myeloencephalitis
}

\author{
Timothy J. Cunningham ${ }^{1, *}$, Jeffrey Greenstein ${ }^{2}$ and Lihua Yao ${ }^{1}$ \\ ${ }^{I}$ Department of Neurobiology and Anatomy Drexel University College of Medicine, Philadelphia, Pennsylvania, \\ Philadelphia, PA 19129, USA \\ ${ }^{2}$ The Multiple Sclerosis Research Institute, Philadelphia, PA, 19146, USA
}

\begin{abstract}
Catalytic sites of metal dependent phosphatases and phospholipases are characterized by a catalytic histidine (H) and adjacent acidic metal binding residue E (or D). For the secreted phospholipases A2 (sPLA2s), the active sites are stabilized by nearby flanking cystines. CHEC-9 (CHEASAAQC), which displays this organization, is a sPLA2 inhibitor that is an effective treatment for models of traumatic and autoimmune neurodegenerative disorders. We screened modifications of this sequence and found that elimination of the two central alanines followed by cyclization results in another potent sPLA2 inhibitor, CHEC-7 (CHEASQC). CHEC-7, like CHEC-9, had uncompetitive kinetic properties making it ideal for in vivo applications. Subcutaneous injections of CHEC-7 into restrained rats inhibited the transient rise in plasma sPLA2 activity suggesting that this peptide was also effective in vivo. Oral delivery of the peptide attenuated the LPSinduced increases in sPLA2 activity. Thin layer chromatography and mass spectroscopy of representative LPS-treated rats suggested that the peptide also reduced levels of lysophosphatidylcholine, a hydrolytic product of the enzyme reaction. In order to test the efficacy of CHEC-7 for neuroimmune therapies, rats were immunized with spinal cord homogenates to induce experimental autoimmune encephalomyelitis (EAE), followed by daily treatment with oral $(1.5 \mathrm{mg} / \mathrm{kg})$ or subcutaneous $(0.1,0.5$, and $1.5 \mathrm{mg} / \mathrm{kg}$ ) CHEC-7 and CHEC-9 ( $\mathrm{n}=10$ for each different condition). The rats were scored for severity of disease applying a standard EAE scale. Control rats all developed disease over the 23-day course of the experiments. Both CHEC-9 and CHEC-7 produced highly significant reductions in disease severity including via oral delivery. The performance of oral CHEC-7 was notable in that this treatment completely prevented disease in half the rats. The results suggested that continued study of modifications of CHEC sequences would provide stable therapeutic peptides for a variety of inflammatory and autoimmune disorders.
\end{abstract}

\section{INTRODUCTION}

The secreted phospholipase A2 enzymes (sPLA2s) represent unique therapeutic targets because enzyme activity is associated directly with both inflammatory and cell death mechanisms. Since the enzymes are present and active in the circulation, it is not surprising that there has been a significant effort aimed at designing sPLA2 inhibitors for systemic treatment of a number of disorders [1]. Several of the effective inhibitors identified so far are of the competitive type containing the indole analogue Me-Indoxam, and are targeted toward specific sPLA2 isoforms [2]. The inhibitors may also have nonenzymatic anti-inflammatory effects by blockade of the sPLA2 M-receptor [3]. However, the performance of these molecules in disease models has been variable and clinical success very limited [1, 4-6]. We identified serendipitously a nine amino acid internal fragment of cell survival/anti-inflammatory protein DSEP, and found that this peptide was an inhibitor of systemic sPLA2 activity $[7,8]$. The peptide, called CHEC-9, is broad spectrum because it inhibits the diverse enzyme activities in the plasma of rats and humans, dominated by groups II and $\mathrm{X}$ sPLA2s, as well as purified enzymes in groups I and III.

*Address correspondence to this author at the Department of Neurobiology and Anatomy Drexel University College of Medicine, 2900 Queen Lane, Philadelphia, PA 19129 USA; E-mail: tcunning @drexelmed.edu
Kinetic studies showed that the peptide is unique among sPLA2 inhibitors, operating with uncompetitive properties. It is dependent therefore on the levels of substrate and on enzyme activity. In theory, such inhibitors should be well suited to in vivo models especially those with an inflammatory component because there is the potential for cascading effects in which active sPLA2 enzymes and their substrates accumulate [8-10]. In fact, systemic treatment with CHEC-9 is effective in reducing SPLA2 and cell-mediated inflammation, neuron death, and behavioral deficits when applied to in vivo models, including trauma and autoimmune disease (experimental autoimmune encephalomyelitis, EAE) [7, 11].

The sequence of CHEC-9 (CHEASAAQC) is interesting because it contains residues that are typical of enzyme catalytic sites in general [12]. The HE (or HD) motif is found consistently in active sites of phosphatases and phospholipases and represents a critical participant in catalysis [7, 13]. Also like CHEC-9, catalytic regions of the secreted isoforms of the PLA2s have flanking cystines presumably for stabilization of the active site. It is therefore not surprising that CHEC-9 was identified as an sPLA2 inhibitor given that peptide-based inhibitors are often derived from key motifs in the target molecules (e.g. ref [14]). In addition, the fact that the peptide is active systemically after subcutaneous application suggested that this molecule must have other properties that optimize its stability and unencumbered transport to its targets in vivo. In testing modifications of the CHEC-9 sequence, we sought initially to determine the biological 
activity of a simple economical modification of CHEC-9 by removing the two central alanines to give CHEASQC (CHEC-7). This modification preserved the basic organization (the HE motif, the flanking cross linked cystines) but changed the hydrophilicity and effective radius of the cyclic peptide. Since there is considerable current interest in oral formulations of disease modifying drugs for MS [15], we also investigated and compared the feasibility and efficacy of oral delivery of the CHECs, comparing both the original and modified versions of the peptide. We found that CHEC-7 was a potent SPLA2 inhibitor, also uncompetitive, and that both CHEC-7 and CHEC-9 were active after oral delivery.

\section{MATERIALS AND METHODS}

\section{Subjects}

Female rats of both the Sprague-Dawley (enzyme studies) and Lewis (EAE studies) strains were obtained from Harlan for use in these experiments. Pooled human blood samples were obtained from Bioreclamation, Inc. All specific procedures of this study were approved by both the Institutional Animal Care and Use Committee and by Institutional Review Board of Drexel University.

\section{Enzyme Assays}

The assays were conducted at ambient temperature $\left(22-25^{\circ} \mathrm{C}\right)$ using a Victor 3 fluorescent reader (Perkin Elmer). The sources of secreted phospholipase A2 were either purified enzymes, group Ia (Naja mossambica venom) or group Ib (porcine pancreatic), both obtained from Sigma, or the plasma of rats and humans. For the rat experiments, blood was either obtained from the trunk after decapitation, or for the timed studies, via tail nick after placing the rats in a standard restrainer. Blood samples were treated with citrate-phosphate-dextrose anticoagulant (1:10, Sigma), and plasma prepared by centrifugation, before freezing at $-80^{\circ}$ until used in the assays. Substrates included 1,2-Bis(1-pyrenebutanoyl)-sn-glycero-3-phosphocholine (Invitrogen) or 1-Palmitoyl-2-Pyrenedecanoyl Phosphatidylcholine (Caymen Chemical), substrates for all calcium dependent PLA2s with the exception of cPLA2 and PAF-AH. These substrates form phospholipids vesicles in aqueous solutions and produce fluorescent pyrenyldecanoic acid upon hydrolysis, which was measured at $350 \mathrm{~nm}$ excitation, $405 \mathrm{~nm}$ emission. Another fluorogenic PLA2 substrate, N- ((6-(2,4-dinitrophenyl) amino) hexanoyl)-2-(4,4-difluoro-5,7-dimethyl-4-bora-3a,4adiaza-s-indacene-3-pentanoyl)-1-hexadecanoyl-sn-glycero-3phosphoethanolamine, triethylammonium salt (PED6, Invitrogen), was also used. The substrates were dissolved in ethanol, and stored at $-20^{\circ}$ prior to use. Mixed substrates were also prepared in which the pyrene-PCs were mixed in the organic phase with equimolar phosphatidic acid (Avanti Polar Lipids) and dispersed in reaction buffer (see below). Mixed liposomes were prepared using Lipofast (Avestin, Inc.), monitored in a fluorescent microscope, and further dispersed until uniform by freeze-thaw cycles. All substrate solutions were prepared in reaction buffer consisting of $50 \mathrm{mM}$ tris ( $\mathrm{pH}=7.4), 0.1 \mathrm{M} \mathrm{NaCl}, 2 \mathrm{mM} \mathrm{CaCl} 2,0.25 \%$ fatty acid-free albumin (Sigma) just prior to use. Kinetic parameters including the properties of CHEC peptides were determined by measuring the initial velocities (Vo) of enzyme reactions (within 2 minutes of initiation). For experiments in which active sPLA2 enzyme concentration was measured in plasma samples from treated rats, we used a single substrate concentration and measured the steady-state rate of the enzyme reaction for 60 minutes. This rate is proportional to the concentration of active enzyme in the plasma if product formation during this period is linear with respect to time [8]. Enzyme activities were expressed relative to baseline or vehicle treatment, or for kinetic studies, fluorescent units (RFU) were converted to product concentration using a pyrenyldecanoic acid (Invitrogen) standard curve. All points on these graphs presented in results were means from 2-3 reactions in individual experiments which were repeated 3 or more times with the same result, i.e., the shapes of the product curves and the direction of change of $\mathrm{Km}$ and Vmax were the same following inhibitor treatment. $\mathrm{Km}$ and Vmax and $\mathrm{r} 2$ were determined with regression software (Prism) and statistical calculations with INSTAT both from Graphpad (San Diego, CA).

\section{D Thin Layer Chromatography and Mass Spectroscopy}

These procedures are described in detail in a previous study [16]. Briefly, lipids were extracted from plasma samples using the Folch method and total phospholipids (PL) were determined. Samples were mixed with a fixed amount of $\mathrm{N}$-Acetyl-L-erythro-sphingosine as an internal loading control, dried under nitrogen, and re-suspended in chloroform before spotting on Merck 5631 TLC plates. The plates were developed in 3 directions with drying between each migration [17]. Solvents for the first and second migration were 1) 60:30:8:2, chloroform: methanol: formic acid: H20, and 2) 50:40:7:3, chloroform: methanol: $28 \%$ ammonium hydroxide, respectively. For the third migration the plates were developed in diethyl ether. After development, the plates were immersed in $10 \% \quad \mathrm{CuSO}_{3}$ and $8 \% \mathrm{H}_{3} \mathrm{PO}_{4}$ solution for 20 seconds, and charred at $180^{\circ} \mathrm{C}$ for $20 \mathrm{~min}$. For mass spectroscopy, samples from the Folch-extracted lipid were dried and re-dissolved in chloroform-methanol-300 $\mathrm{mM}$ ammonium acetate in water $(60: 133: 7, \mathrm{v} / \mathrm{v} / \mathrm{v})$. Lysophosphatidylcholines were analyzed by electrospray ionization triple quadrupole mass spectrometry at the Kansas Lipidomics Research Center, as previously described [18].

\section{Peptide Preparation and Delivery}

CHEC-7 (CHEASQC) and CHEC-9 (CHEASAAQC) were synthesized as linear peptides by Celtek, Inc. The peptides were cross-linked in tris-buffered saline ( $\mathrm{pH}$ 7.5) at $0.25 \mathrm{mg} / \mathrm{ml}$ overnight. Free sulfhydryls were measured using Ellman's reagent and were undetectable after incubation. Cross-linking was verified by electrospray mass spectrometry as in previous studies [7]. Peptide concentrations were adjusted for delivery of stated dosages either subcutaneously under the loose skin of the back or p.o. by gavage. Control animals received an equivalent volume of vehicle.

\section{Lipopolysaccharide (LPS) Treatment and EAE Immunization}

LPS $(4 \mathrm{mg} / \mathrm{kg})$ was administered subcutaneously under the skin of the upper thigh. For the EAE studies, Mycobacterium tuberculosis H37Ra (MT, Difco) was crushed with 
pestle and mortar and used to enrich Complete Freund's Adjuvant (CFA, Sigma) at a final concentration of $10 \mathrm{mg} / \mathrm{ml}$. Lyophilized Guinea Pig Spinal Cord Homogenate (Sigma) was weighed and suspended in PBS to yield $40 \mathrm{mg} / \mathrm{mL}$. The homogenate was mixed with equal amount of CFA/MT and emulsified prior to injection into the right hind paw of each rat at a volume of $0.1 \mathrm{ml}$.

\section{Evaluation of the EAE Clinical Signs}

We used a standard EAE scoring paradigm: 0- Normal behavior, no clinical signs; 1-tail weakness, tail was limp and droops; 2- Hind-legs were hypotonic and weak; paresiswobbly walk, hind legs unsteady or one hind-leg was dragged; 3- hind-legs paralyzed, front legs normal; 4 hindlegs paralyzed, front legs weak; 5- full paralysis. Experimentally blinded observers, experienced in EAE studies, scored the rats.

\section{Statistical Analysis}

Nonparametric statistical tests including Mann-Whitney test and Kruskal-Wallis ANOVA were used throughout the study as indicated.

\section{RESULTS}

\section{Inhibition of Plasma sPLA2 Activity}

CHEC-7 was screened in vitro using concentrations between 1 and $100 \mathrm{nM}$ applied to reactions of groups Ia, Ib (both at $20 \mathrm{nM}$ ), and of rat (10\%) and human (40\%) plasma all with the pyrene substrates. CHEC-9 was used as a positive control in most experiments. CHEC-7 inhibited all these sources of sPLA2, most effectively at peptide concentrations under $25 \mathrm{nM}$ and substrate concentrations above $30 \mu \mathrm{M}$. CHEC-7 inhibition is illustrated in product curves (side by side with CHEC-9), and in a representative Michaelis-Menten plot using human plasma and a range of substrate concentrations (Fig. 1A, B). Both sets of data were consistent with properties of an uncompetitive inhibitor. For the product curves (Fig. 1A), the small delay initially in the inhibition by the peptides allows for the formation of the enzyme-substrate (ES) complex, which is targeted by uncompetitive inhibitors. Thereafter, the decline of activity persisted for the entire experiment, apparently because of stable binding by the inhibitor to this complex [8]. Furthermore, the data suggested that both CHEC-7 and CHEC-9 have similar potencies ex vivo, both operating at the low nanomolar range for human plasma. CHEC-7 was also effective at nanomolar concentrations for the other sPLA2s tested (data not shown).

Human and rat plasma displayed classical MichaelisMenten kinetics but $\mathrm{Km}$ varied from sample to sample, also depending on the particular substrate used in the reactions. Therefore, the range of substrate and plasma concentrations that demonstrated these kinetic properties was determined empirically. Substrates consisting of mixed liposomes containing 10-Pyrene PC and phosphatidic acid (PA) yielded robust activity in reactions with human and rat plasma, perhaps because of the preference of group II sPLA2 enzymes for PA [19]. These reactions generated classical Michaelis-Menten curves with substrate concentrations between $20-90 \mu \mathrm{M}$. This allowed identification of the properties of CHEC-7 inhibition by nonlinear regression of these curves (Fig. 1B). This analysis showed the reduction in both Vmax and Km, the hallmark of an uncompetitive inhibitor (legend Fig. 1). The curves also showed no CHEC-7 inhibi-

\section{Human Plasma sPLA2 Activity}

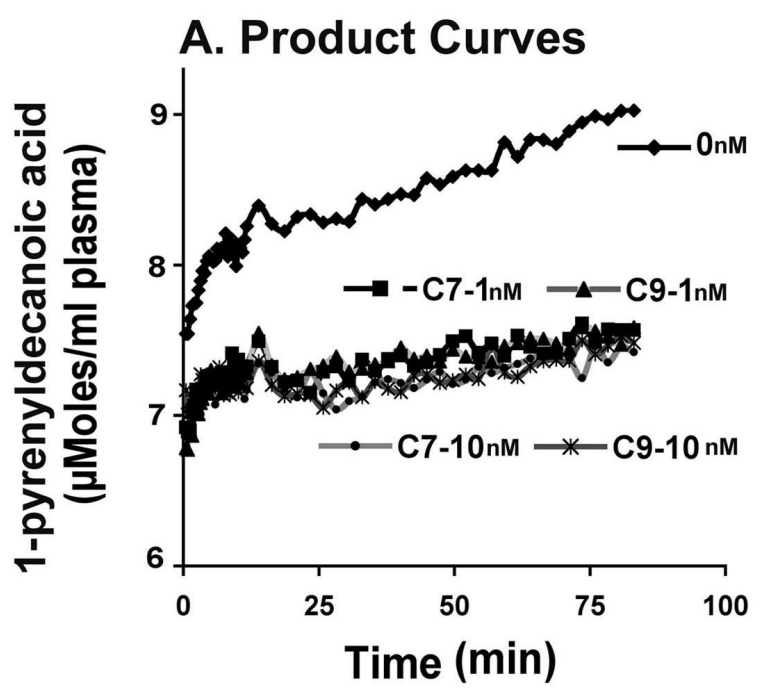

B. Michaelis-Menten Plot

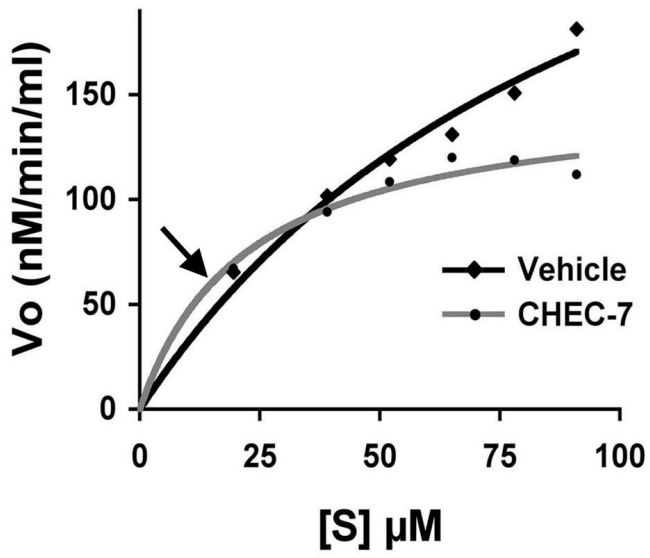

Fig. (1). CHEC-7 was uncompetitive inhibitors of SPLA2s in human plasma. A. Enzyme reactions of 20\% human plasma and $80 \mu \mathrm{M} 1$ hexadecanoyl-2-(1-pyrenedecanoyl)-sn-glycero-3-phosphocholine with and without CHEC-7 and CHEC-9 at 1 and 10nM. At this substrate concentration, the inhibition was evident early in the reaction and stable for the entire time course of the experiments. B. Michaelis-Menten plots of Vo versus substrate concentration for human plasma reacted with equimolar phosphatidic acid- pyrenyl PC mixed liposomes (see methods) in the presence of $5 \mathrm{nM}$ CHEC-7. Note the initially exaggerated velocity with peptide addition to reactions containing low substrate concentrations (arrow) and then increased efficiency of the inhibition as substrate concentrations increase. These properties as well as the kinetic parameters determined by nonlinear regression of these curves show that the inhibition is uncompetitive. [Vmax: vehicle $=366.9$, CHEC-7=150.9 Kd: vehicle $=104.7$, CHEC-7=22.5. Ki $=13.1 \pm 6.9 \mathrm{nM}$, calculated using substrate concentrations $>40 \mu \mathrm{M}]$. 
tion of enzyme activity (in fact exaggerated activity) at low substrate concentrations, again consistent with the inhibitor's affinity for the ES complex. Finally, we also found inhibition of both plasma and purified sPLA2s (Ia, Ib) in reactions with a phosphoethanolamine base substrate PED6 at $20-40 \mu \mathrm{M}$ using nanomolar CHEC-7 concentrations (data not shown). Thus, the broad-spectrum activities found previously with CHEC-9 were also evident for CHEC-7.

\section{CHEC-7 Treatment of Rats Inhibits Plasma SPLA2} Activity In Vivo

\section{Simple Restraint Model}

In previous studies, we documented a transient rise in plasma sPLA2 activity within the first two hours after restraint in a standard rat holder [8]. This rise was eliminated by subcutaneous injections of CHEC-9, given $30 \mathrm{~min}$ after initiating the restraint. In this study, we repeated these experiments with CHEC-7. Subcutaneous injections of CHEC-7 also eliminated the transient increase in plasma sPLA2 activity of restrained rats (Fig. 2), suggesting the peptide was active in vivo after systemic administration.

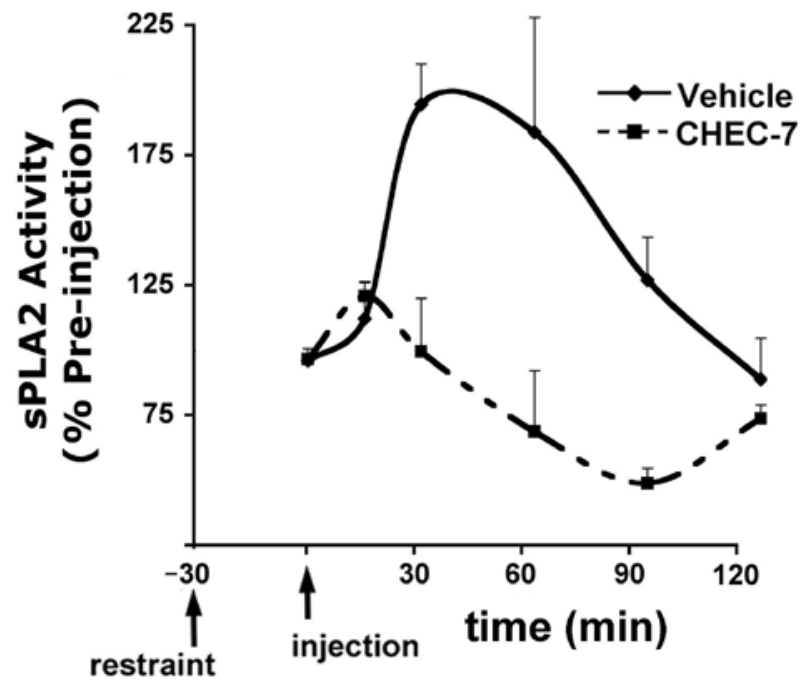

Fig. (2). Reduced levels of active SPLA2 enzyme in rat plasma samples after systemic delivery of CHEC-9. A transient rise in mean levels of active enzyme was found in the first $2 \mathrm{hrs}$ following placement of the animal in a standard rat restrainer. Subcutaneous CHEC-7 inhibits this increase $30 \mathrm{~min}$ after initiating restraint inhibits this increase. Each data point represents the mean \pm s.d. of 3 rats (2-3 measurements/time pt./rat) in each of the two treatment groups expressed as a percent of the pre-injection sPLA2 levels. The inhibition by peptide treatment is significant $(\mathrm{p}=0.0041$, Kruskal-Wallis ANOVA).

\section{Oral Delivery of CHEC-7: Lipopolysaccharide (LPS) Model}

We tested the efficacy of oral delivery of CHEC-7 by comparing sPLA2 activity levels in plasma of peptide and vehicle treated rats after treatment with LPS. Gavage of peptide or vehicle solution was $1 \mathrm{hr}$. after the LPS injection and plasma sPLA2 activity was determined $2 \mathrm{hrs}$ later. The timing of these experiments was based on published data in rat showing the maximum febrile response to subcutaneous
LPS [20]. The data in Fig. (3A) was from a range of dosages from $.2 \mathrm{mg} / \mathrm{kg}$ to $1.5 \mathrm{mg} / \mathrm{kg}$ as we found no apparent dose effect in this range.

We conducted a preliminary examination of plasma lysophosphatidylcholine (lysoPC) after lipids were extracted from the plasma of rats injected with LPS. Figs. (3B,C). The rats were treated with $0.5 \mathrm{mg} / \mathrm{kg}$ peptide, vehicle, or untreated $(n=2$ each condition). The data from these samples suggests that LysoPC levels were elevated after LPS treatment and maintained (or returned) close to normal with peptide treatment. Interestingly, the mass spectroscopy experiments, which reveal specific lysoPC species based on composition of the A1 fatty acid, suggest that not all lysoPCs responded similarly to LPS, and that peptide treatment reduced only the elevated species. This behavior was consistent with the activity dependent properties of CHEC peptides suggested in this and previous studies $[8,11]$.

\section{CHEC-7 Inhibits EAE After Immunization with Spinal Cord Homogenates}

In the present investigation, guinea pig spinal cord was used to immunize Lewis rats, a model which typically results in more severe disease than that used in previous studies with CHEC-9 [11]. Therefore, both CHEC-7 and CHEC-9 were tested in this model, with the peptides applied daily following immunization, either by subcutaneous injections or by oral delivery. Both the injected and orally treated rats showed highly significant inhibition of disease activity with peptide compared to controls. The results for orally treated rats are shown in Fig. (4A). There were no significant differences or trends in the data when mean disease scores for CHEC-7 and CHEC-9 treated rats (in this or any treatment group) are compared. When CHEC-7 and CHEC-9 were also compared for overall disease prevention (Fig. 4B), fewer CHEC-7 treated rats displayed disease than CHEC-9 treated rats, although this difference failed to reach significance. The results did show however that disease inhibition makes a significant contribution to the lower of disease scores in the peptide-treated rats, especially those treated with CHEC-7.

\section{DISCUSSION}

CHEC-9 was identified originally as a fragment of a cell survival protein that was isolated from oxidatively-stressed neural cells and has also been identified as a survivalpromoting molecule by other laboratories [21-25]. The peptide is located in the $\mathrm{N}$-terminus of this molecule and was synthesized originally to prevent cell death in various neurodegenerative models [21]. The further identification of the peptide as sPLA2 inhibitor with anti-inflammatory effects was consistent with its cell survival-promoting properties [8]. Since the peptide was effective after systemic administration, it is logical to suggest that it could be developed for clinical use for several conditions in which PLA2 enzymes are implicated and have the potential to be destructive. Part of this effort includes identifying modifications of the peptide that are both economical and effective. Like CHEC-9, CHEC-7 spontaneously cyclized at physiological $\mathrm{pH}$, providing some conformational and metabolic stability [26]. With that achieved, dropping the 2 central alanines was economical in terms of effective radius of the cyclic peptide. There is also an increase average hydrophilicity, a key element influencing oral bioavailability of highly 


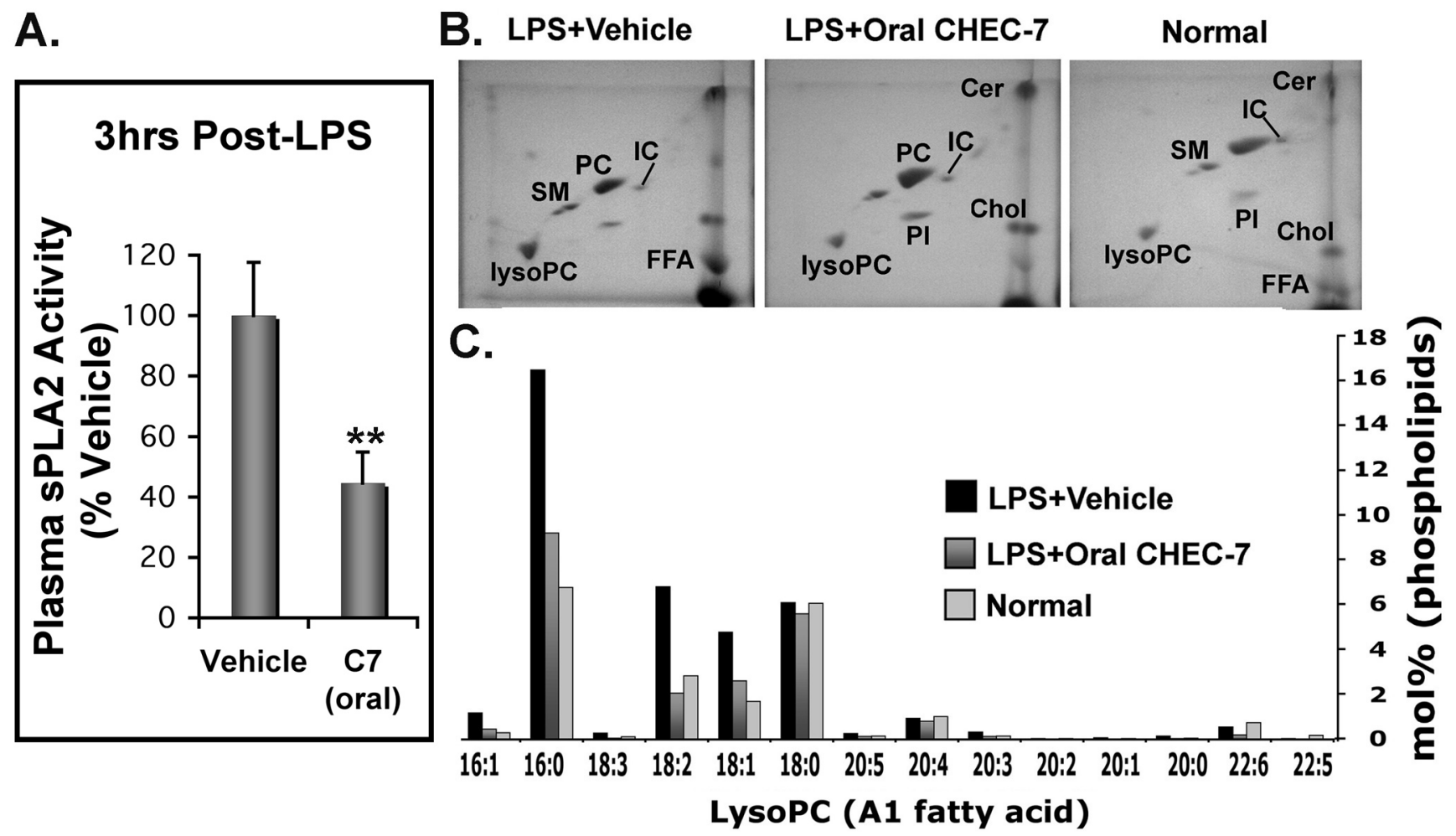

Fig. (3). Oral treatment with CHEC-7 inhibits plasma sPLA2 activity after lipopolysaccharide (LPS) injections. A. Inhibition of plasma sPLA2 activity by CHEC-7 is expressed as a percent of the average values obtained for vehicle treated rats. A range of oral CHEC-7 dosages $(0.2-1.5 \mathrm{mg} / \mathrm{kg})$ was effective without a significant dose effect in this range. $($ Mean + s.d., Vehicle $=100+17.6 \%$, $\mathrm{n}=6$, CHEC-7 $=44.5+10.3 \%, \mathrm{n}=8,2$ each @ 0.2, 0.5, 0.75, $1.5 \mathrm{mg} / \mathrm{kg}), \mathrm{p}=0.0041$, Mann Whitney. B. Thin layer chromatography showing the lysoPC spots relative to other abundant plasma lipids in rats receiving the $0.5 \mathrm{mg} / \mathrm{kg}$ dose of oral CHEC-7. A prominent LysoPC spot is found in the vehicle-treated rats. Abbreviations: Cer-ceramide; PC- phosphatidylcholine; SM- sphingomyelin; PI- phosphatidylinositol. Chol- free cholesterol; FFA- free fatty acids; LysoPC- lysophosphatidylcholine. InCer- internal control. C. Mol percent of individual species of lysoPCs in the $0.5 \mathrm{mg} / \mathrm{kg}$ samples calculated from mass spectra of plasma lipid extracts relative to total phospholipids present in the samples. Note that the response of different LysoPCs to LPS and peptide treatment was not uniform.

soluble peptides like the CHECs (see below). At the same time CHEC-7 preserves the critical HE sequence and $\mathrm{pI}$ the larger peptide.

\section{Specificity}

The emphasis in the present study on inhibition of plasma sPLA2 activity allowed the data to be related to potential efficacy for in vivo models after systemic treatments. Although the number of different sPLA2 isoforms active in the plasma and tissues of rats and humans is diverse, the key property of the CHEC peptides, one that contributes significantly to their in vivo efficacy, may just be their lack of specificity. Thus for rat plasma, PLA2 activity and peptide inhibition were readily demonstrated using a phosphatidylcholine substrates targeted by all sPLA2s, but not cPLA2 (cytosolic) or LP-PLA2 (lipoprotein-associated PLA2). Human plasma showed optimal significant activity to a modified mixed pyrenePC-phosphatidic acid substrate as well as to a phosphatidylethanolamine substrate (PED 6) that apparently has no restriction as to PLA2 isoforms. The CHEC inhibitors then are presumed to target a number of sPLA2s, perhaps depending more on the affinity of these enzymes for their substrates, rather than the specific sPLA2 isoform (see below). This is in contrast to the highly specific inhibitors that were developed to neutralize a particular
sPLA2s by blocking the active site, most commonly that of sPLA2 group IIA [1, 4-6]. However, the in vivo and clinical efficacy of these compounds has been disappointing. CHECs have consistently been very effective for in vivo models, particularly autoimmune and traumatic models of neurodegeneration. It is likely therefore that several PLA2 isoforms contribute to these disorders and broad coverage is most effective.

The fact that the CHEC peptides as uncompetitive inhibitors may, on the other hand, dictate some other variety of specificity of action. As uncompetitive inhibitors, CHEC peptides bind the enzyme-substrate complex, and so it is likely that both elements of the ES target contribute to the peptide's inhibitory activity. At the very least, our data suggest that the inhibition depends on enzyme activity (which in turn depends on formation of the ES complex), so the substrate preferences of sPLA2 enzymes may be critical for the stable binding of these peptides. One reason that uncompetitive inhibitors are so rare [10] is that detection of this type of inhibition may indeed require correct identification of both enzyme and substrate. A number of potentially important enzyme inhibitors may have been overlooked because typical screening procedures generally don't vary the substrates as systematically as they do possible enzymes targets. 

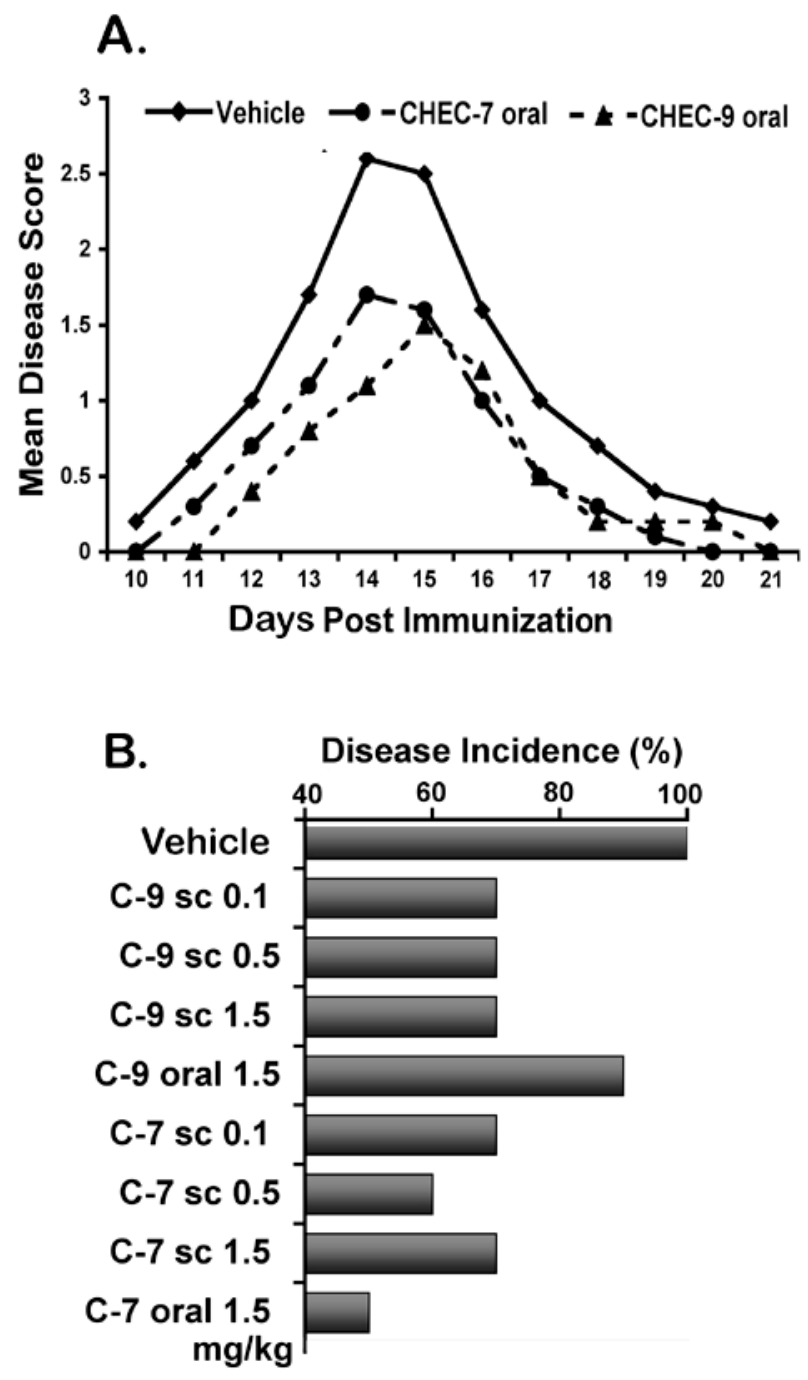

Fig. (4). Disease scores and incidence for rats treated with CHEC-7 and CHEC-9 peptides in guinea pig spinal cord/CFA experimental autoimmune encephalomyelitis (EAE) model. A. Disease scores following daily oral doses of CHEC-7 and CHEC-9 $(1.5 \mathrm{mg} / \mathrm{kg})$ were compared to vehicle treated rats $(\mathrm{n}=10$ for each condition). The data were highly significant for both peptides comparing days when significant disease activity was evident (days 12-19, $\mathrm{p}<10^{-4}$, Kruskal-Wallis ANOVA). Similar significant differences in disease activity were obtained for all subcutaneous dosages (data not shown). B. Disease incidence in all groups of rats expressed as a percent of the 10 animals in each treatment arm. Vehicle treated rats all showed some disease, while 1-5 out of the 10 rats in each of the peptide-treated groups were disease free. CHEC-7 treated animals, on average, had lower disease incidence than CHEC-9 treated animals but this difference between thee two peptides failed to reach significance ( $\mathrm{p}=0.063$, Mann Whitney).

\section{Oral Delivery}

In a previous study, CHEC-9 delivered by subcutaneous injections to successfully treat traumatic CNS injury and experimental autoimmune encephalomyelitis (EAE). In the present study, we also tested oral peptide delivery because of high current interest in this treatment option for Multiple
Sclerosis [15]. Oral treatment was successful. However, at this time we have no information on the transit of the CHEC peptides into the circulation from any site of peptide delivery. Nonetheless, it is feasible that a very stable hydrophilic peptide will cross the intestinal mucosa barrier by diffusion along the paracellular route (i.e., between the epithelial cells). The net charge on both CHEC-7 and CHEC-9 is -1, which may be ideal for such transport [27]. However, the key element governing transport is size, which should put CHEC-7 at an advantage [28]. Overall, in terms of disease prevention, there may have been an advantage with CHEC-7, although verification of differences in efficacy may require additional subjects. Pharmakinetic and pharmacodynamic studies with these peptides are in progress and may also help settle this issue. Nonetheless, the present study suggests that continued study of modifications of the basic CHEC sequence motif will provide additional stable therapeutic peptides that can be applied to a number of systemic and neurodegenerative disorders, especially those with a strong inflammatory component.

\section{ACKNOWLEDGEMENTS}

We would like to thank Mary Roth and Ruth Welti of the Kansas Lipidomics Research Center for mass spectrometric analysis of the lysophosphatidylcholines. The Kansas Lipidomics Research Center is supported by National Science Foundation (EPS 0236913, MCB 0455318, DBI 0521587), Kansas Technology Enterprise Corporation, K-IDeA Networks of Biomedical Research Excellence (INBRE) of National Institute of Health (P20RR16475), and Kansas State University. This work was also supported by grants from the Craig Nielsen Foundation and the Amyotrophic Lateral Sclerosis Association to TJC.

\section{CONFLICT OF INTEREST}

Drexel University, T.J. Cunningham and Lihua Yao have obtained or filed for patent protection of the peptides used in this study.

\section{REFERENCES}

[1] Springer DM. An update on inhibitors of human $14 \mathrm{kDa}$ Type II s-PLA2 in development. Curr Pharm Des 2001; 7(3): 181-98.

[2] Smart BP, Pan YH, Weeks AK, Bollinger JG, Bahnson BJ, Gelb MH. Inhibition of the complete set of mammalian secreted phospholipases A2 by indole analogues a structure-guided study. Bioorg Med Chem 2004; 12(7): 1737-49.

[3] Boilard E, Rouault M, Surrel F, et al. Secreted phospholipase A 2 inhibitors are also potent blockers of binding to the m-type receptor. Biochemistry 2006; 45(44): 13203-18.

[4] Abraham E, Naum C, Bandi V, et al. Efficacy and safety of LY3 $315920 \mathrm{Na} / \mathrm{S}-5920$, a selective inhibitor of $14-\mathrm{kDa}$ group IIA secretory phospholipase A2, in patients with suspected sepsis and organ failure. Crit Care Med 2003; 31(3): 718-28.

[5] Bradley JD, Dmitrienko AA, Kivitz AJ, et al. A randomized, double-blinded, placebo-controlled clinical trial of LY333013, a selective inhibitor of group II secretory phospholipase A2, in the treatment of rheumatoid arthritis. J Rheumatol 2005; 32(3): 417-23.

[6] Zeiher BG, Steingrub J, Laterre PF, Dmitrienko A, Fukiishi Y, Abraham E. LY315920NA/S-5920, a selective inhibitor of group IIA secretory phospholipase A2, fails to improve clinical outcome for patients with severe sepsis. Crit Care Med 2005; 33(8): 1741-8.

[7] Cunningham $\mathrm{T}$, Souayah N, Jameson B, Mitchell J, Yao L. Systemic treatment of cerebral cortex lesions in rats with a new secreted phospholipase A2 inhibitor. J Neurotrauma 2004; 21(11): 1683-91. 
[8] Cunningham TJ, Maciejewski J, Yao L. Inhibition of secreted phospholipase A2 by neuron survival and anti-inflammatory peptide CHEC-9. J Neuroinflamm 2006; 3(1): 25.

[9] Westley AM, Westley J. Enzyme Inhibition in open systems. J Biol Chem 1996; 271(10): 5347.

[10] Cornish-Bowden A. Why is uncompetitive inhibition so rare? A possible explanation, with implications for the design of drugs and pesticides. FEBS Lett 1986; 203(1): 3-6.

[11] Cunningham T, Yao L, Oetinger M, Cort L, Blankenhorn E, Greenstein J. Secreted phospholipase A2 activity in experimental autoimmune encephalomyelitis and multiple sclerosis. J Neuroinflamm 2006; 3: 26.

[12] Bartlett GJ, Porter CT, Borkakoti N, Thornton JM. Analysis of catalytic residues in enzyme active sites. J Mol Biol 2002; 324(1): 105-21.

[13] Barford D. Molecular mechanisms of the protein serine/threonine phosphatases. Trends Biochem Sci 1996; 21(11): 407-12.

[14] Ruggeri ZM, Houghten RA, Russell SR, Zimmerman TS. Inhibition of platelet function with synthetic peptides designed to be high-affinity antagonists of fibrinogen binding to platelets. Proc Natl Acad Sci USA 1986; 83(15): 5708-12.

[15] DeAngelis T, Lublin F. Multiple sclerosis: new treatment trials and emerging therapeutic targets. Curr Opin Neurol 2008; 21(3): 261-71.

[16] Cunningham TJ, Yao L, Lucena A. Product inhibition of secreted phospholipase A2 may explain lysophosphatidylcholines' unexpected therapeutic properties. J Inflamm 2008; 5: 17.

[17] Yokoyama K, Shimizu F, Setaka M. Simultaneous separation of lysophospholipids from the total lipid fraction of crude biological samples using two-dimensional thin-layer chromatography. J Lipid Res 2000; 41(1): 142-7.
[18] Bartz R, Li WH, Venables B, et al. Lipidomics reveals that adiposomes store ether lipids and mediate phospholipid traffic. $\mathrm{J}$ Lipid Res 2007; 48(4): 837-47.

[19] Snitko Y, Yoon ET, Cho W. High specificity of human secretory class II phospholipase A2 for phosphatidic acid. Biochem J 1997; 321(Pt 3): 737-41.

[20] Luheshi GN. Cytokines and fever. Mechanisms and sites of action Ann N Y Acad Sci 1998; 856: 83-9.

[21] Cunningham TJ, Hodge L, Speicher D, et al. Identification of a survival-promoting peptide in medium conditioned by oxidatively stressed cell lines of nervous system origin. J Neurosci 1998; 18(18): 7047-60.

[22] Landgraf P, Sieg F, Wahle P, Meyer G, Kreutz MR, Pape HC. A maternal blood-borne factor promotes survival of the developing thalamus. FASEB J 2005; 19(2): 225-7.

[23] Lowrie AG, Wigmore SJ, Wright DJ, Waddell ID, Ross JA Dermcidin expression in hepatic cells improves survival without $\mathrm{N}$-glycosylation, but requires asparagine residues. $\mathrm{Br} \mathrm{J}$ Cancer 2006; 94(11): 1663-71.

[24] Schittek B, Hipfel R, Sauer B, et al. Dermcidin: a novel human antibiotic peptide secreted by sweat glands. Nat Immunol 2001; 2(12): 1133-7.

[25] Porter D, Weremowicz S, Chin K, et al. A neural survival factor is a candidate oncogene in breast cancer. Proc Natl Acad Sci USA 2003; 100(19): 10931-6.

[26] Li P, Roller PP. Cyclization strategies in peptide derived drug design. Curr Top Med Chem 2002; 2: 325-41.

[27] Borchard RT, Jeffrey A, Siahaan TJ, Gangwar S, Pauletti GM. Improvement of oral peptide bioavailability: Peptidomimetics and prodrug strategies. Adv Drug Deliv Rev 1997; 27(2-3): 235-56.

[28] Pauletti GM, Gangwar S, Knipp GT, et al. Structural requirements for intestinal absorption of peptide drugs. J Control Release 1996; 41(1-2): 3-17. 